\title{
Patch Departure Behavior of Bumble Bees: Rules and Mechanisms
}

\author{
Dale E. Taneyhill \\ Yupparaj Wittayarlai, Amper Muang, Chiang Mai 50100, Thailand \\ Correspondence should be addressed to Dale E. Taneyhill, detaneyhill@hotmail.com
}

Received 1 August 2009; Accepted 6 February 2010

Academic Editor: Koos (J. C.) Biesmeijer

Copyright () 2010 Dale E. Taneyhill. This is an open access article distributed under the Creative Commons Attribution License, which permits unrestricted use, distribution, and reproduction in any medium, provided the original work is properly cited.

\begin{abstract}
I present an increment-decay model for the mechanism of bumble bees' decision to depart from inflorescences. The probability of departure is the consequence of a dynamic threshold level of stimuli necessary to elicit a stereotyped landing reaction. Reception of floral nectar lowers this threshold, making the bee less likely to depart. Concurrently the threshold increases, making departure from the inflorescence more probable. Increments to the probability of landing are an increasing, decelerating function of nectar volume, and are worth less, in sequence, for the same amount of nectar. The model is contrasted to threshold departure rules, which predict that bees will depart from inflorescences if the amount of nectar in the last one or two flowers visited is below a given level. Field tests comparing the two models were performed with monkshood (Aconitum columbianum). Treated flowers contained a descending series of nectar volumes ( 6 to $0 \mu \mathrm{L}$ of $30 \%$ sucrose solution). The more nectar that bees encountered in the treated flowers, the more likely they were to remain within the inflorescence after subsequently visiting one to three empty flowers. I discuss the differences between rules and mechanisms in regard to cognitive models of foraging behavior.
\end{abstract}

\section{Introduction}

For the majority of flowering plants, successful reproduction depends on a mutualism with insect pollinators. As with all such coevolved interactions, the two parties are motivated by self-interest: the plant is provided with an efficient means of pollen transfer, while the pollinator receives energy and nutrients in the form of floral nectar and pollen. The fine details of this interaction include factors such as the number of flowers that the pollinator should visit within an inflorescence before departing and moving to another plant of the same species. Plants should maximize the amount of pollen exported to the stigmas of conspecifics, while simultaneously minimizing the level of geitonogamous selfpollination. The pollinator, meanwhile, should behave so as to maximize its net rate of energy gain, and should stay within an inflorescence until it is more profitable to depart and move to another. Pollinator departure from inflorescences thus falls within the scope of patch departure in foraging theory, a central sub-discipline of behavioral ecology [1].

Evolutionary study of patch departure began with the marginal value theorem (MVT) [2], which specifies how foragers should exploit patches in order to maximize the long-term net rate of energy gain. However, the MVT itself does not specify a realistic departure rule or policy $[3,4]$. This is due to the MVT's assumption of complete information: the forager is in effect omniscient, knowing all the relevant data about a patch before entering it. Proximal or "cognitive" models of patch departure should produce roughly the same decisions as would the MVT, while making realistic use of available environmental cues.

Theoretical work has shown how information gained while foraging within patches can be used to construct an optimal departure policy $[3,5,6]$. A general, flexible formalism makes use of Bayes' Theorem to derive optimal departure rules given various distributions of the number of prey within patches [3,7-11]. Graphical representations of several such models are shown in Figure 1. Following Stephens and Krebs, I refer to these models as increment-decay processes, due to the continuous dynamics of the expected remaining patch time [12]. These models are interesting for reasons other than just the possibility of stochastic optimization. Notably, the incrementing and decrementing dynamics have neurobiological analogues, in processes such as habituation 


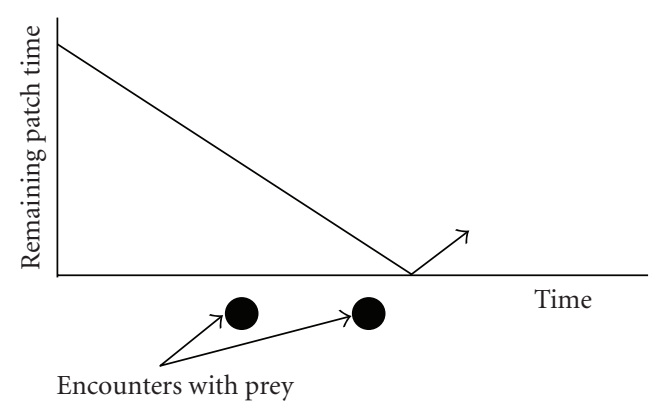

(a) Poisson

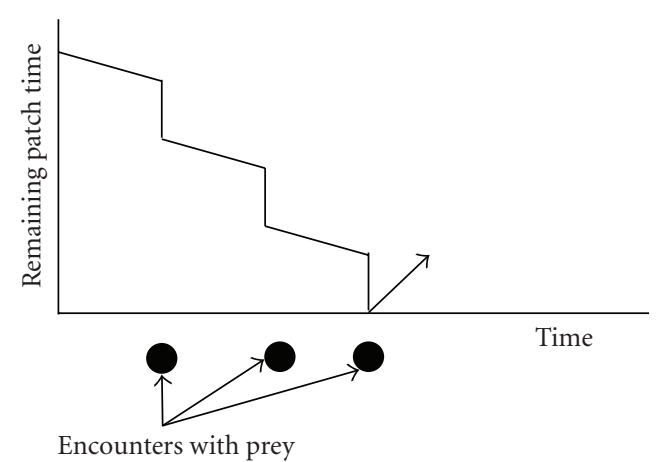

(b) Binomial

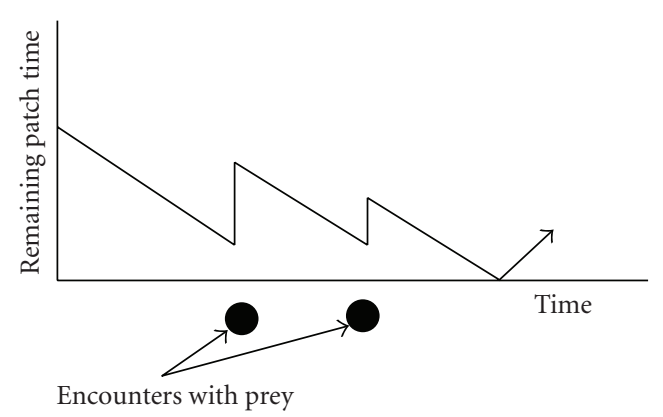

(c) Negative binomial

FIGURE 1: Graphical Representations of several Bayesian patch departure rules $[7,12]$, for three distributions of the number of prey within patches. The ordinate represents expected remaining time in the patch. Solid circles represent encounters with prey. (a) For the Poisson distribution, prey captures yield no information and departure occurs after a fixed time independent of the number of prey encountered. (b) For the binomial distribution, each prey capture decrements expected remaining patch time. (c) For the negative binomial, each prey capture increments expected remaining patch time, but each increment in sequence is smaller.

and sensitization to stimuli. Waage [13] first suggested that the parasitic wasp Venturia (=Nemeritis) canescens uses an increment-decay process for searching and departing from patches of its host.

One can see that, in outline, an increment-decay model could describe the behavior of bumble bees or other nectarivores visiting multiple-flowered plants. An inflorescence can be considered as a patch, and each encounter with a prey (in this case, a flower that contained nectar) would affect the expected remaining number of flowers to be visited on the plant before departing. However, such models have only very rarely been explored in regard to bee foraging behavior [14-16]. The reason is that departure from inflorescences can often be analyzed via a discrete version of the MVT that applies to the situation of overlapping encounters, meaning that the forager meets more than one prey at a time $[17,18]$. Many plants have an inflorescence in which the flowers are arranged vertically on a central stem, blooming sequentially from the bottom upward. As a result, flowers near the bottom often contain more nectar than others higher on the stem [19]. To maximize the long-term net rate of energy gain, bees should begin at the bottom of such an inflorescence and work upward. At some point the diminishing amounts of nectar in higher flowers would make it more profitable to depart the inflorescence and move to another plant. We should then observe that bees often depart from such vertical inflorescences before visiting all available flowers, and this has been observed in the field [19-22] and in the laboratory [23]. Thus a number of authors have proposed the following "threshold departure rule": the bee should depart the inflorescence when

$$
E(S)<E(L)
$$

where $E(S)$ (stay) is the expected profitability from the next flower visited within the current inflorescence, and $E(L)$ (leave) is the expected profitability from the first flower visited after departure [24-28].

The original threshold departure rules are now generally thought to be too simplistic a description of patch departure in bumble bees $[1,14,15]$. In this paper I present an increment-decay model, similar to those that have been proposed for parasitoids, but specifically tailored to bumble bees. I present the results of field experiments designed to contrast the model's predictions with those of the threshold departure rules.

\section{The Model}

The increment-decay model for patch departure presented here was originally applied to bumble bee foraging at a higher level, the situation in which there is patchiness in nectar within large meadows of the relevant plant species, but no discrete patches as such [14]; it was soon realized that a similar model in outline could apply to departure behavior within inflorescences.

The first assumption is that bumble bees land on inflorescences and begin to probe the flowers for nectar if the set of stimuli presented by the inflorescence are sufficient to release a stereotyped behavioral pattern, hereafter called the landing reaction [29]. In vector notation, let $\mathbf{s}$ be a vector of stimulus intensities from various modalities, including visual, olfactory, and tactile, and let $\mathbf{w}$ represent a vector of weightings for each $s_{i} \in \mathbf{s}$; the scalar product $z=\mathbf{s}^{\mathrm{T}} \mathbf{w}$ defines a real number $z$ that is mapped onto a decision function $f(z)$ that returns the conditional probability of landing given $z$, 
$P($ Land $\mid z)$. The simplest form of $f(z)$ would be the step function

$$
f(z)= \begin{cases}1, & z \geq z^{*}, \\ 0, & z<z^{*},\end{cases}
$$

where $z^{*}$ is a threshold level necessary to evoke the landing behavior. In (2) the response is all or none for fixed $z$; modification to make the response probabilistic may easily be done by making $f(z)$ a sigmoid function:

$$
f(z)=\frac{1}{1+\lambda \exp \left(-\beta\left(z-z^{*}\right)\right)} .
$$

Equation (3) approaches a step function in the limit as $\beta \rightarrow \infty$. Since (2) and (3) return a conditional probability, we may use for $f(z)$ any function that is also a distribution function of a random variable; thus the requirements are that $f(z)$ is right-continuous, is nondecreasing, approaches 0 in the limit as $z \rightarrow-\infty$, and approaches 1 in the limit as $z \rightarrow \infty$

The above statements are an extremely simplified description of a cognitive system that integrates sensory information and initiates behavioral output. Note that the weightings to various stimuli presented by the flowers may include highly negative weightings, making the bee less likely to land; such stimuli include scent marks left by other bees, holes in nectar-robbed flowers, or marks on flower petals made by bees' tarsal claws. In addition, the measures of and the weightings to these stimuli may be both dynamic and varied among individuals. In other words, the inputs $s_{i} \in \mathbf{s}$ and the weights $w_{i} \in \mathbf{w}$ are not assumed to be fixed, but may change due to learning, or may vary due to individual differences among bees: for example, visual accuracy can vary with bee head size [30].

The core assumption of the model is that the threshold parameter $z^{*}$ (or equivalently, the function $f(z)$ itself) is a dynamic variable, continually changing with time and in the light of recent experience. The probability that a bee will probe the next flower or will depart the inflorescence thus depends on how $f(z)$ changes with time and experience. Finding nectar causes $z^{*}$ to decrease, making the bee more likely to probe the next flower, and thus incrementing the expected remaining time within the inflorescence. Concurrently, I assume that $z^{*}$ spontaneously increases, so that the expected remaining time within the inflorescence decays between nectar encounters.

The second assumption is that there are maximum and minimum values of $z^{*}$, and these are reflecting boundaries. The minimum is automatically reflecting since $z^{*}$ increases continually. Upon reaching its maximum, I assume that the threshold then lowers until the next nectar encounter; if the bee has departed the inflorescence at that point, the probability of flower stimuli evoking the landing reaction increases monotonically during interplant travel. Thus the longer the distance to the next plant, the lower will be the threshold $z^{*}$ upon encountering the next inflorescence. This means that bees will tend to stay longer within inflorescences the greater the average distance between plants. This is

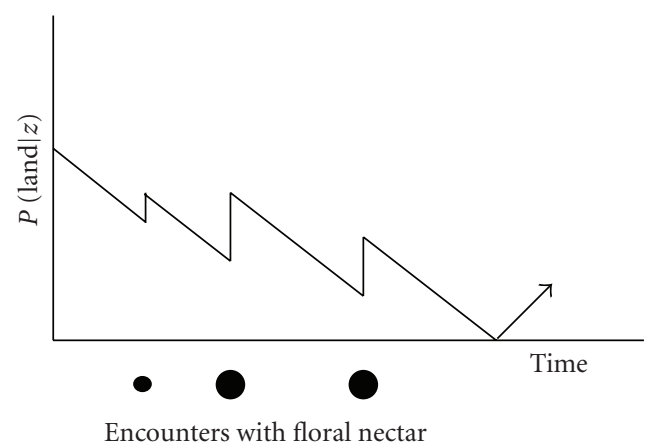

FIGURE 2: A graphical interpretation of the increment-decay model for bumble bee patch departure. The conditional probability of landing on the next flower given the weighted stimuli sum $z, P$ (Land $\mid z)$, is plotted as a function of time. The probability declines monotonically with time. If the bee finds nectar within a flower, the probability increases in proportion to the nectar volume. Solid circles represent reception of nectar, with the radius proportional to the nectar volume. Note that for the same amount of nectar the amount of incrementing is less in sequence, following (4) in the text. When the threshold $z^{*}$ reaches its maximum value, it then spontaneously decreases, increasing the landing probability during interpatch travel (arrow).

a fundamental prediction of the MVT, here produced via a mechanism similar to that proposed by Ollason [31].

The above assumptions can be interpreted graphically as an increment-decay process (Figure 2). Each reception of nectar (solid circles) decreases $z^{*}$, thus increasing the probability of landing on the next flower, increasing the expected remaining patch time by an amount proportional to the volume of nectar. Concurrently, $z^{*}$ increases between nectar encounters, meaning that the expected remaining time within the inflorescence decays.

The dynamics of expected remaining patch time resemble the potential function of McNamara [6], with several important differences. Here the processes of decay and increment affect a threshold response to stimuli, with the result that the effects of nectar reception may be spread across several discrete patches, for example, flower groupings within the same plant or on adjacent plants $[32,33]$. In the model the decision to depart is influenced by possibly many attributes of inflorescences besides the standing crops of nectar. This explains, for example, the observation by Pyke that bees stay longer within larger inflorescences of Aconitum columbianum even when these contain no more nectar than smaller inflorescences [19]. Larger inflorescences will have a greater value of $z$, all other things being equal.

Several points should be clarified concerning the decay process. In Figure 2 the rate of decay is constant for illustrative purposes, but in general it may be nonlinear, following an exponential or hyperbolic trajectory, for example. The increments are shown happening instantaneously, as in other such models (Figure 1); in reality each flower visit includes a handling time. We may keep the convenient form shown by assuming that the increments represent the net effect of increase due to nectar minus decay incurred during the time of the flower visit. This allows for the possibility that a flower 
visit may actually result in a net decrement in expected remaining patch time, if the handling time for that flower is long enough. In addition, the model to this point does not explicitly specify the probability of departure from the whole inflorescence, which may be less than 1 given that the bee does not land (i.e., bees may reject the current flower, but stay within the same inflorescence). The conditional probability of departure given $z$ may be specified by any nonnegative function of $z$ bounded above by $1-P($ Land $\mid z)$.

Reception of energy in the form of floral nectar lowers the threshold $z^{*}$, thus incrementing the expected remaining patch time. In the formal Bayesian foraging models cited above, the increments from prey capture have been constant or limited to a small number of values $[3,8,10]$. In the current model the "prey" is a continuous variable, an amount of energy depending on a volume of nectar and the nectar sugar concentration. The change in $z^{*}$ will be given by a function $\phi$ that has as arguments the volume of nectar (given a constant sugar concentration) and other information, such as the order of encounter of flowers within the inflorescence. I assume that $\phi=\phi(V, k)$ is a function of at least two arguments, the volume of nectar $V$ in the flowers, and the order of their encounter within the inflorescence, $k$. I make the following assumptions for the form of $\phi(V, k)$ :

$$
\frac{\partial \phi}{\partial V}>0, \quad \frac{\partial^{2} \phi}{\partial V^{2}}<0, \quad \frac{\partial \phi}{\partial k}<0,
$$

where $V$ is the volume of nectar and $k=1,2, \ldots n$ is the order of encounter, with $k=1$ being the first flower encountered after the threshold has attained its maximum and thus begun to decrease. The first two partial derivatives mean that increments are an increasing and decelerating function of the amount of nectar. This is simply the familiar WeberFechner Law for the subjective scaling of stimuli (see [3437] for further application of the Weber Law to amount in foraging models). The final assumption in (4) states that the increments are a declining function of order; each increment in sequence is worth less for the same amount of nectar, until the threshold $z^{*}$ has reached its maximum.

The evolutionary reasons for these assumptions about the form of $\phi$ follow from the typical distribution of nectar standing crop within and among plants. Often there is a highly clumped distribution, caused by differences in secretion rates between plants or due to local search by the bees themselves $[38,39]$. Responding to occasional bonanzas of nectar within flowers in a linear manner might cause bees to stay much longer than would be optimal within an inflorescence. The decline with order follows from the optimal response of a Bayesian forager when there is a clumped distribution of prey within patches. Iwasa et al. derived optimal Bayesian policies for foragers encountering Poisson, binomial, and negative binomial distributions of prey within patches [7]. An overlooked feature of their equations was that for the negative binomial distribution, which indicates clumping of prey, each increment in sequence is less than the previous increment until the forager has departed the patch (Figure 1(c)). In neurobiology, this decline in the response to stimuli with order of presentation is known as adaptation [40].
The mathematical details in the model are important in distinguishing it from other psychological or constrained optimality models for patch departure. The major difference concerns a horizon effect: the "time window" over which past experience affects departure decisions may appear to change. For example, decisions in one patch may be influenced by experience in previously visited patches if the bee departed from those earlier patches before the threshold $z^{*}$ had reached its maximum. If the latter occurs, however, the bee's behavior in the current patch may appear to be a function of the last travel distance only. This difference in the apparent time window provides direct contrast with threshold departure and other "run of bad luck" (ROBL) models. In a one-flower threshold departure rule the decision to depart at each flower is independent of experience at previous flowers, while in the general ROBL model there is always a specified time or number window beyond which past experience has little or no bearing on present decisions. Similar arguments apply to general memory window rules of the Linear Operator Model (LINOP) or exponentially weighted moving average (EWMA) forms [4, 41, 42]. All such models employ a weighting of events within the time window to predict current decisions. A heavy weighting to more recent experience can explain the often observed strong effect of last travel distance on patch departure [42]. However, this weighting then predicts that foragers will rapidly adapt to changes in conditions such as average interpatch travel time, which is usually not the case.

The proposed model can explain the results of diverse experiments with bumble bees. Thomson et al. observed that bumble bees departed from empty umbels of Aralia hispida after visiting two flowers [32]. After leaving an umbel that had been enriched by placing $0.5 \mu \mathrm{L}$ sucrose solution into all 12 open flowers, the bees probed an average of five flowers on a subsequent empty umbel before departing. On the enriched umbels, bees visited an average 14 flowers before departing, meaning that they revisited two flowers. These revisited flowers presumably contained the strongly negative stimulus of a marking pheromone, meaning that $z$ for recently visited flowers will be lower than that for a simply empty flower. To be consistent with the model, other bees arriving at such recently visited flowers would probe fewer than two on average before departing: they would either visit one-flower or not land on the umbel at all; this behavior has been noted in other experiments [26, 43-45]. After departing from the enriched umbel, if the threshold $z^{*}$ remained lowered due to the reception of nectar, there would then be a carryover effect on the ensuing empty umbel.

In a set of laboratory experiments with artificial umbels, Taneyhill and Thomson observed that bumble bees probed approximately the same number of flowers in umbels containing either 2 or $4 \mu \mathrm{L}$ of nectar in each of eight artificial flowers, but fewer in umbels containing $1 \mu \mathrm{L}$ in each flower [33]. For the increment-decay model, this would suggest that there was little difference in the value of $\phi$ for 2 and $4 \mu \mathrm{L}$ due to its decelerating form, but that the effect of $1 \mu \mathrm{L}$ was significantly less. When bees visited sets of three artificial 
umbels, one empty and the other two containing two of the above three volumes of nectar, the numbers of flowers probed within the empty umbels depended on both the nectar volumes in previously visited umbels and their order of encounter. The bees probed almost identical numbers of flowers within empty umbels after first visiting either an umbel filled with 2 or $4 \mu \mathrm{L}$ in each flower, and also nearly identical numbers after visiting umbels with both amounts in either order. However, they probed more after a 2 than a $1 \mu \mathrm{L}$ umbel, and more after first a 2 and then a $1 \mu \mathrm{L}$ umbel than vice versa. These results were consistent with the assumption that each increment is worth less, in sequence, for the same value of $V$ : the asymmetry occurs because $(\phi(2, k)+\phi(1, k+$ $1))>(\phi(1, k)+\phi(2, k+1))$. If 4 and $2 \mu \mathrm{L}$ are interchangeable, however, then $(\phi(4, k)+\phi(2, k+1))=(\phi(2, k)+$ $\phi(4, k+1))$, and thus the order of presentation makes no difference.

Cresswell also studied bumble bees foraging from ringlike inflorescences, in wild bergamot Monarda fistulosa [26]. He obtained estimates of departure probability as a function of nectar volume by finding the amount of nectar that was just sufficient to make bees always stay, recording the probability of departure from empty flowers, and drawing a line between the two in a plot of departure probability as a function of nectar volume. When values from this function were used in computer simulations, the predicted numbers of flowers visited consistently overestimated the actual numbers visited in study plots. This can easily be explained by the increment decay model due to its assumptions that departure probability increases continually and that each increment with nectar is worth less in sequence. In the stochastic threshold departure rule, departure probabilities remain the same at each flower for the same nectar volume.

Formal parameterizations of the model will be unique to each experimental system. In the field, the important flower stimuli and their relative weightings in the landing decision can be determined by statistical techniques such as principal components analysis, as Cresswell and Robertson have done for Campanula rotundifolia [46]. Recently much progress has been made in the analysis of how stimuli such as marking pheromones affect bees' decisions to exploit patches, and how such reactions are blended with experience and variation within individuals $[47,48]$.

The increment decay model makes several robust predictions that can be tested in comparison to the threshold departure rules. A deterministic, one-flower threshold departure rule predicts that bumble bees will always depart an inflorescence after finding an empty flower, since the threshold volume must be equal to or greater than zero. The analogous stochastic rule predicts that bees will depart after finding an empty flower with a fixed probability independent of experience at other flowers. The increment decay model, however, predicts that departure probability at empty flowers will decrease with increasing nectar in previously encountered flowers. The same arguments may be applied to two-flower and similar departure rules. I thus tested the two alternative models in the field, using one of the field systems that spawned the original threshold departure rules.

\section{Methods}

Field work was done in the vicinity of the Rocky Mountain Biological Laboratory, Gothic, Colorado. The protocol for the field experiments was to remove nectar from flowers within plants with vertical inflorescences and to place known amounts of sucrose solution (in all experiments I used reagent grade sucrose, $30 \mathrm{~g}$ solute per $100 \mathrm{~mL}$ distilled water) into flowers that bees would be likely to visit prior to the empty flowers. Since bumble bees often forage from plants with vertical inflorescences by starting near the bottom and moving upward [20,21], I placed the sucrose solution into the bottom flowers in all experiments, and drained the second or second and third flowers from the bottom of all floral nectar.

The experiments reported here were done using monkshood, Aconitum columbianum. The monkshood inflorescence has dark purple, zygomorphic flowers borne on a central spike, with two cup-shaped nectaries hidden inside a hood-shaped cap formed by the fusion of two petals (see [49] for illustrations of how bees forage from monkshood). This species is well suited to tests of the threshold departure rules, because the structure of the flower makes it difficult for bees to sense the presence of nectar before landing and probing the flower, and the nectar can easily be removed from the nectaries within the flowers.

The first experiment tested one-flower threshold departure rules. I used 4 nectar treatments for the bottom flowers: $4,2,1$, and 0 (=control) $\mu \mathrm{L}$ of $30 \%$ sucrose solution. The largest amount was chosen because this was approximately the highest standing crop volume found in surveys of the study populations (sugar concentration of samples was usually higher, generally in the range of $40 \%$ to $50 \%$ sucrose equivalents, so the treatments actually contained less sugar). I placed these amounts of nectar into the bottom flowers of study plants and drained the flower above the bottom of all the nectar. Although it would be easy to ensure that empty flowers contained no nectar at all by removing the nectaries, I did not do this for fear of altering the bees' behavior. Instead I checked the flowers for nectar every 20 minutes using $1 \mu \mathrm{L}$ microcapillary tubes. Hamilton dispensing microsyringes were used to place the sucrose solution into the flowers. Observations were made with 6 plants on each observation day, and I used plants with at least 6 open flowers on the inflorescence. Plants were not selected at random; rather they were chosen from those that had nectaries in very good condition. While the bees visiting the plants were not marked, due to identification of caste and species it was determined that a minimum of 6 individual bees visited the plants.

I recorded the pattern of visitation, time of visit, caste, and species for all bees visiting the test plants. Because I found it difficult to accurately record data for all five nectar amounts at the same time, each day of observation was devoted to one nectar volume only; data for each replication of each treatment are thus analyzed separately.

A second experiment with A. columbianum tested both one- and two-flower threshold departure rules. I used as nectar treatments $6,4,2,1$ and $0 \mu \mathrm{L} 30 \%$ sucrose solution. 
TABle 1: Departure patterns of bumble bees on treated inflorescences of Aconitum columbianum. Four microliters of $30 \%$ sucrose solution were placed in bottom flowers of the inflorescence, with zero microliters (empty) in second-from-bottom flowers. The notation in this and all subsequent tables is $X, 2$ standing for bees that began by visiting the empty second flower first, without visiting the bottom flower. 1, 2 stands for bees visiting the treated bottom flower and then the empty second flower. Goodness of fit test for the null hypothesis of equal probability to depart: $\chi^{2}=4.67, P<.01$.

\begin{tabular}{lccc}
\hline & Stay & Depart & Probability (Depart) \\
\hline$X, 2$ & 13 & 6 & 0.32 \\
1,2 & 19 & 1 & 0.05 \\
\hline
\end{tabular}

TABle 2: Departure patterns of bumble bees on treated inflorescences of Aconitum columbianum. Second replication of the experiment with four microliters of $30 \%$ sucrose solution placed in bottom flowers of the inflorescence, zero in second-from-bottom flowers. Goodness of fit test for the null hypothesis of equal probability to depart: $\chi^{2}=5.24, P<.01$.

\begin{tabular}{lccc}
\hline & Stay & Depart & Probability (Depart) \\
\hline$X, 2$ & 11 & 5 & 0.31 \\
1,2 & 22 & 1 & 0.04 \\
\hline
\end{tabular}

TABLE 3: Departure patterns of bumble bees on treated inflorescences of Aconitum columbianum. Two microliters of $30 \%$ sucrose solution placed in bottom flowers of the inflorescence, zero in second-from-bottom flowers. $\chi^{2}=1.73, P>.05$.

\begin{tabular}{lccc}
\hline & Stay & Depart & Probability (Depart) \\
\hline$X, 2$ & 22 & 10 & 0.31 \\
1,2 & 19 & 3 & 0.15 \\
\hline
\end{tabular}

I drained the second and third flowers from the bottom of all nectar, and again checked these every 20 minutes using microcapillary tubes. I used a Rainin digital dispensing pipet to place sucrose solution into the bottom flowers. During this experiment, I was able to gather data for all five amounts of sucrose on each day of observation. I changed the amount of sucrose in each study plant every two hours during each day. Bee density in this experiment was greater than for the first experiment; at least 10 individuals were visiting the experimental plants.

Data from the first experiment were cast into $2 \times 2$ contingency tables and analyzed via two-way tests of independence, with the null hypothesis that the probability of departure from an empty flower was independent of previous experience (i.e., whether the bee had first visited the treated flower). Data from the second experiment were analyzed via an $R \times C$ contingency table test [50].

\section{Results}

In tests of one-flower threshold departure rules using Aconitum columbianum, bumble bees' probability of departure from empty flowers decreased with increasing volume of nectar in previously encountered bottom flowers (Tables $1,2,3,4,5,6$, and 7 ). With $4 \mu \mathrm{L}$ of sucrose solution
TABLe 4: Departure patterns of bumble bees on treated inflorescences of Aconitum columbianum. Second replication of two microliters of $30 \%$ sucrose solution placed in bottom flowers of the inflorescence, zero in second-from-bottom flowers. $\chi^{2}=3.95, P<$ .05 .

\begin{tabular}{lccc}
\hline & Stay & Depart & Probability (Depart) \\
\hline$X, 2$ & 31 & 17 & 0.35 \\
1,2 & 24 & 4 & 0.14 \\
\hline
\end{tabular}

TABle 5: Departure patterns of bumble bees on treated inflorescences of Aconitum columbianum. One microliter of $30 \%$ sucrose solution placed in bottom flowers of the inflorescence, zero in second-from-bottom flowers. $\chi^{2}=0.269, P>.5$.

\begin{tabular}{lccc}
\hline & Stay & Depart & Probability (Depart) \\
\hline$X, 2$ & 21 & 5 & 0.19 \\
1,2 & 19 & 3 & 0.14 \\
\hline
\end{tabular}

TABle 6: Departure patterns of bumble bees on treated inflorescences of Aconitum columbianum. Second replication of one microliter of $30 \%$ sucrose solution placed in bottom flowers of the inflorescence, zero in second-from-bottom flowers. $\chi^{2}=3.72$, $P<.06$.

\begin{tabular}{lccc}
\hline & Stay & Depart & Probability (Depart) \\
\hline$X, 2$ & 33 & 7 & 0.18 \\
1,2 & 31 & 1 & 0.03 \\
\hline
\end{tabular}

TABLe 7: Departure patterns of bumble bees on treated inflorescences of Aconitum columbianum. Control treatment with zero microliters of $30 \%$ sucrose solution in bottom flowers of the inflorescence, and zero in second-from-bottom flowers. $\chi^{2}=$ $1.63, P>.1$.

\begin{tabular}{lccc}
\hline & Stay & Depart & Probability (Depart) \\
\hline$X, 2$ & 25 & 14 & 0.36 \\
1,2 & 10 & 8 & 0.44 \\
\hline
\end{tabular}

in bottom flowers, the bees almost never departed after subsequently finding the empty flower if they had first visited the treated flower (probabilities of departure $=0.04$ and 0.05 for two replicate experiments). In the control treatment with both flowers empty, the probability of departure from the empty second flower was greater if the bee had first visited the treated (empty) bottom flower, as is predicted by the increment-decay model, although the two probabilities were not statistically different. The probability of departure from the empty second flower, given that it was visited first, was about 0.3 (overall $P=.29, N=220$ ), and in each experiment was fairly close to this value except for the two experiments using $1 \mu \mathrm{L}$. Observations collected on different days showed nearly identical patterns, except for the second $1 \mu \mathrm{L}$ treatment.

Results of the two-flower tests (Tables 8 and 9) were in accord with the one-flower experiments. The probabilities of departure depended on both the amount of nectar in the treated bottom flower and the pattern of visitation (3-way test of independence using a log-linear model; $G=28.085$ 
TABLE 8: Departure patterns of bumble bees on treated inflorescences of Aconitum columbianum. Control treatment with zero microliters of $30 \%$ sucrose solution in bottom flowers of the inflorescence, and zero in second-from-bottom flowers (2), and zero in third-from-bottom flowers (3). The notation in this and Table 9 is $X, 2$ or 3 stands for bees that began by visiting the empty second or third flower first, without visiting the bottom flower. $X$, 2 and 3 stands for bees visiting both empty flowers first. 1, 2, or 3 stands for visiting the treated bottom flower and then the empty second or empty third flower. 1, 2 and 3 stands for visiting the treated bottom flower and then both the empty second and empty third flowers.

\begin{tabular}{lccc}
\hline & Stay & Depart & Probability (Depart) \\
\hline$X, 2$ or 3 & 65 & 27 & 0.29 \\
$X, 2$ and 3 & 14 & 6 & 0.30 \\
1,2 or 3 & 20 & 1 & 0.048 \\
1,2 and 3 & 8 & 1 & 0.11 \\
\hline
\end{tabular}

TABLE 9: Departure patterns of bumble bees on treated inflorescences of Aconitum columbianum. The experiment used a descending series of volumes of $30 \%$ sucrose solution, from 6 to 0 microliters, in bottom flowers of the inflorescence, with secondfrom-bottom and third-from-bottom flowers empty. The notation for visit patterns is as detailed in Table 8. Three-way log-likelihood test of independence, $G=28.085, P<.01$ for the 3-way interaction.

\begin{tabular}{|c|c|c|c|}
\hline & Stay & Depart & Probability (Depart) \\
\hline \multicolumn{4}{|l|}{$6 \mu \mathrm{L}$} \\
\hline$X, 2$ or 3 & 38 & 12 & 0.24 \\
\hline$X, 2$ and 3 & 2 & 4 & 0.67 \\
\hline 1,2 or 3 & 30 & 0 & 0 \\
\hline 1,2 and 3 & 11 & 0 & 0 \\
\hline \multicolumn{4}{|l|}{$4 \mu \mathrm{L}$} \\
\hline$X, 2$ or 3 & 33 & 17 & 0.34 \\
\hline$X, 2$ and 3 & 7 & 2 & 0.22 \\
\hline 1,2 or 3 & 55 & 1 & 0.018 \\
\hline 1,2 and 3 & 33 & 4 & 0.11 \\
\hline \multicolumn{4}{|l|}{$2 \mu \mathrm{L}$} \\
\hline$X, 2$ or 3 & 30 & 17 & 0.36 \\
\hline$X, 2$ and 3 & 3 & 4 & 0.57 \\
\hline 1,2 or 3 & 38 & 2 & 0.5 \\
\hline 1,2 and 3 & 20 & 3 & 0.13 \\
\hline \multicolumn{4}{|l|}{$1 \mu \mathrm{L}$} \\
\hline$X, 2$ or 3 & 26 & 11 & 0.3 \\
\hline$X, 2$ and 3 & 7 & 2 & 0.22 \\
\hline 1,2 or 3 & 49 & 4 & 0.08 \\
\hline 1,2 and 3 & 21 & 3 & 0.125 \\
\hline \multicolumn{4}{|l|}{$0 \mu \mathrm{L}$} \\
\hline$X, 2$ or 3 & 97 & 43 & 0.31 \\
\hline $\mathrm{X}, 2$ and 3 & 25 & 9 & 0.265 \\
\hline 1,2 or 3 & 52 & 13 & 0.20 \\
\hline 1,2 and 3 & 22 & 7 & 0.24 \\
\hline
\end{tabular}

$P<.01$ for the 3-way interaction). As in the one-flower study, the probabilities of departure from the empty second flowers decreased with increasing amounts of sucrose placed in the bottom flower. With $6 \mu \mathrm{L}$ sucrose solution placed in the bottom flower, the bees never departed after next probing either one or two empty flowers. The probabilities of departure from empty second or third flowers visited first were again about 0.3 , as in the one-flower experiments (overall, $P=.31, N=324$ ), with relatively little variation across treatments (range, 0.24 to 0.36 ).

\section{Discussion}

The results from field experiments with Aconitum columbianum strongly suggest that the bumble bees did not use a one- or two- flower threshold departure rule. In many cases the bees did not depart after visiting from one to three flowers that were, to the limit of the experimental techniques, empty of nectar. The probabilities of departure from empty flowers were instead influenced by the amounts of nectar in previously encountered flowers, meaning that the bees were also not using a stochastic threshold departure rule. When testing threshold departure rules one could continue to advocate them with an increasing number window; in the present case the results do not falsify a three-flower stochastic threshold departure rule. However, this line of reasoning could be extended indefinitely. To interpret the results of Thomson et al. [32] in that manner, bees visiting umbels of Aralia hispida would have to have been switching between a two-flower and a five-flower stochastic threshold departure rule. It seems much more parsimonious to think of expected departure time or number as being flexible, able to vary with time and experience.

In this paper I have presented the increment-decay process as a general framework for understanding patch departure in bumble bees. Such a model, in which patch departure is considered as an observable aspect of a dynamical system, addresses the important question of how cognitive mechanisms of foraging behavior should be considered in theory above the neurobiological level. The terms "rules" and "mechanisms" are perhaps considered interchangeable by some, yet I argue that there can be subtle but important differences between the two. The word "rule" often denotes the type of if-then procedure found in expert computer systems or other such human constructs; many such rules have been proposed in foraging theory, including give-up time rules, fixed number rules, failure rules, and the threshold departure rules $[3,8,24,51]$. However, other proposed mechanisms, such as those described in the present paper, cannot easily be expressed in this form. An everpresent danger in building cognitive models of behavior is that they run the risk of assigning to the animal processes that may account for the observations, but which may not in fact exist. Representing mechanisms as dynamical systems may bring the models closer to the underlying neurobiology while sacrificing ease of understanding them in commonsense terms.

Constructing cognitive models for patch departure behavior in this manner (adding details of mechanism peculiar to each organism) means that the results become further removed from the MVT [4]. What, then, is the purpose of the optimality models? The standard answer is 
that they help build intuition about the mechanisms that one expects to find $[52,53]$. The model presented in this paper was suggested by observations of bees and by the results of experiments. However, the approach was guided by principles from optimality theory, in both the overall structure of the model and in its assumptions concerning how experiences affect the model's dynamics. Incrementdecay processes appear to be a fortunate compromise between the proximal and adaptationist descriptions, having their roots in Bayesian inference while at the same time bearing resemblance to neurobiological processes. Since they have the potential to explain so many aspects of patch departure [11], they should be investigated in depth for bumble bees and other foragers.

\section{Acknowledgments}

The author thanks James Thomson and Lars Chittka for discussion of the ideas presented herein, and the staff of the Rocky Mountain Biological Laboratory, Gothic Co. for field support.

\section{References}

[1] L. Chittka, J. D. Thomson, and N. M. Waser, "Flower constancy, insect psychology, and plant evolution," Naturwissenschaften, vol. 86, no. 8, pp. 361-377, 1999.

[2] E. L. Charnov, "Optimal foraging, the marginal value theorem," Theoretical Population Biology, vol. 9, no. 2, pp. 129-136, 1976.

[3] R. F. Green, "Stopping rules for optimal foragers," American Naturalist, vol. 123, no. 1, pp. 30-43, 1984.

[4] I. A. Todd and A. Kacelnik, "Psychological mechanisms and the marginal value theorem: dynamics of scalar memory for travel time," Animal Behaviour, vol. 46, no. 4, pp. 765-775, 1993.

[5] A. Oaten, "Optimal foraging in patches: a case for stochasticity," Theoretical Population Biology, vol. 12, no. 3, pp. 263-285, 1977.

[6] J. M. McNamara, "Optimal patch use in a stochastic environment," Theoretical Population Biology, vol. 21, no. 2, pp. 269288, 1982.

[7] Y. Iwasa, M. Higashi, and M. Yamamura, "Prey distribution as a factor determining the choice of optimal foraging strategy," American Naturalist, vol. 117, pp. 710-723, 1981.

[8] J. N. McNair, "Optimal giving-up times and the marginal value theorem," American Naturalist, vol. 119, no. 4, pp. 511529, 1982.

[9] R. F. Green, "A simpler, more general method for finding the optimal foraging strategy for Bayesian birds," Oikos, vol. 112, pp. 274-284, 2006.

[10] O. Olsson, "Bayesian foraging with only two patch types," Oikos, vol. 112, no. 2, pp. 285-297, 2006.

[11] J. M. McNamara, R. F. Green, and O. Olsson, "Bayes' theorem and its applications in animal behavior," Oikos, vol. 112, pp. 243-251, 2006.

[12] D. W. Stephens and J. R. Krebs, Foraging Theory, Princeton University Press, Princeton, NJ, USA, 1986.

[13] J. K. Waage, "Foraging for patchily distributed hosts by the parasitoid Nemeritis canescens," Journal of Animal Ecology, vol. 48, no. 2, pp. 353-371, 1979.
[14] D. E. Taneyhill, Evolution of complex foraging behavior in bumble bees, Ph.D. dissertation, State University of New York at Stony Brook, New York, NY, USA, 1994.

[15] D. Lefebvre, J. Pierre, Y. Outreman, and J.-S. Pierre, "Patch departure rules in bumblebees: evidence of a decremental motivational mechanism," Behavioral Ecology and Sociobiology, vol. 61, no. 11, pp. 1707-1715, 2007.

[16] J. M. Biernaskie, S. C. Walker, and R. J. Gegear, "Bumblebees learn to forage like bayesians," American Naturalist, vol. 174, no. 3, pp. 413-423, 2009.

[17] K. D. Waddington and L. Holden, "Optimal foraging: on flower selection by bees," American Naturalist, vol. 114, no. 3, pp. 179-196, 1979.

[18] D. W. Stephens, J. F. Lynch, A. E. Sorensen, and C. Gordon, "Preference and profitability: theory and experiment," American Naturalist, vol. 127, no. 4, pp. 533-553, 1986.

[19] G. H. Pyke, "Optimal foraging in bumblebees: rule of movement between flowers within inflorescences," Animal Behaviour, vol. 27, no. 4, pp. 1167-1181, 1979.

[20] L. S. Best and P. Bierzychudek, "Pollinator foraging on foxglove (Digitalis purpurea): a test of a new model," Evolution, vol. 36, pp. 70-79, 1982.

[21] S. A. Corbet, I. Cuthill, M. Fallows, and T. Harrison, "Why do nectar-foraging bees and wasps work upwards on inflorescences?” Oecologia, vol. 51, no. 1, pp. 79-83, 1981.

[22] G. Pyke, "Optimal foraging in bumblebees: rule of departure from an inflorescence," Canadian Journal of Zoology, vol. 60, pp. 417-428, 1981.

[23] K. D. Waddington and B. Heinrich, "The foraging movements of bumblebees on vertical "inflorescences": an experimental analysis," Journal of Comparative Physiology, vol. 134, no. 2, pp. 113-117, 1979.

[24] C. M. Hodges, "Bumble bee foraging: the threshold departure rule," Ecology, vol. 66, no. 1, pp. 179-187, 1985.

[25] J. M. Pleasants, "Optimal foraging by nectarivores: a test of the marginal-value theorem," American Naturalist, vol. 134, no. 1, pp. 51-71, 1989.

[26] J. E. Cresswell, "How and why do nectar-foraging bumblebees initiate movements between inflorescences of wild bergamot Monarda fistulosa (Lamiaceae)?" Oecologia, vol. 82, no. 4, pp. 450-460, 1990.

[27] R. Kadmon and A. Shmida, "Departure rules used by bees foraging for nectar: a field test," Evolutionary Ecology, vol. 6, no. 2, pp. 142-151, 1992.

[28] J. C. Stout and D. Goulson, "The influence of nectar secretion rates on the responses of bumblebees (Bombus spp.) to previously visited flowers," Behavioral Ecology \& Sociobiology, vol. 52, no. 3, pp. 239-246, 2002.

[29] K. Lunau, "Innate flower recognition in bumblebees (Bombus terrestris, B. lucorum Apidae): optical signals from stamens as landing reaction releasers," Ethology, vol. 88, pp. 203-214, 1991.

[30] J. Spaethe and L. Chittka, "Interindividual variation of eye optics and single object resolution in bumblebees," Journal of Experimental Biology, vol. 206, no. 19, pp. 3447-3453, 2003.

[31] J. G. Ollason, "Learning to forage-optimally?" Theoretical Population Biology, vol. 18, no. 1, pp. 44-56, 1980.

[32] J. D. Thomson, R. C. Plowright, and W. P. Maddison, "Behavior of bumble bee pollinators of Aralia hispida Vent. (Araliaceae)," Oecologia, vol. 54, no. 3, pp. 326-336, 1982.

[33] D. E. Taneyhill and J. D. Thomson, "Behavior of inexperienced bumble bees toward spatial clumping of nectar," Entomologia Generalis, vol. 29, no. 2-4, pp. 149-164, 2007. 
[34] M. Bateson and A. Kacelnik, "Accuracy of memory for amount in the foraging starling, Sturnus vulgaris," Animal Behaviour, vol. 50, no. 2, pp. 431-443, 1995.

[35] M. Bateson and A. Kacelnik, "Preferences for fixed and variable food sources: variability in amount and delay," Journal of the Experimental Analysis of Behavior, vol. 63, no. 3, pp. 313329, 1995.

[36] A. Kacelnik and E. Brito, "Risky choice and Weber's law," Journal of Theoretical Biology, vol. 194, no. 2, pp. 289-298, 1998.

[37] D. E. Taneyhill, "Foraging mechanisms and the currency for models of energy maximization in bumble bees (Hymenoptera: Apidae: Bombus occidentalis)," Entomologia Generalis, vol. 30, no. 2, pp. 119-133, 2007.

[38] D. Brink and J. M. J. deWet, "Interpopulation variation in nectar production in Aconitum columbianum (Ranunculaceae)," Oecologia, vol. 47, no. 2, pp. 160-163, 1980.

[39] M. Zimmerman, "Patchiness in the dispersion of nectar resources: probable causes," Oecologia, vol. 49, no. 2, pp. 154 $157,1981$.

[40] P. R. Killeen, "Mathematical principles of reinforcement," Behavioral and Brain Sciences, vol. 17, no. 1, pp. 105-172, 1994.

[41] A. Kacelnik, J. R. Krebs, and B. Ens, "Foraging in a changing environment: an experiment with starlings (Sturnus vulgaris)," in Quantitative Analyses of Behaviour, M. L. Commons, A. Kacelnik, and S. Shettleworth, Eds., vol. 6 of Foraging, pp. 6387, Lawrence Erlbaum, Hillsdale, NJ, USA, 1987.

[42] I. Cuthill, P. Haccou, and A. Kacelnik, "Starlings (Sturnus vulgaris) exploiting patches-responses to long-term changes in travel time," Behavioral Ecology, vol. 5, no. 1, pp. 81-89, 1994.

[43] J. H. Marden, "Remote perception of floral nectar by bumblebees," Oecologia, vol. 64, no. 2, pp. 232-240, 1984.

[44] M. Giurfa and J. A. Nuñez, "Honeybees mark with scent and reject recently visited flowers," Oecologia, vol. 89, no. 1, pp. 113-117, 1992.

[45] D. Goulson, J. W. Chapman, and W. O. H. Hughes, "Discrimination of unrewarding flowers by bees; direct detection of rewards and use of repellent scent marks," Behavioural Ecology \& Sociobiology, vol. 52, pp. 239-246, 2001.

[46] J. E. Cresswell and A. W. Robertson, "Discrimination by pollen-collecting bumblebees among differently rewarding flowers of an alpine wildflower, Campanula rotundifolia (Campanulaceae)," Oikos, vol. 69, pp. 304-308, 1994.

[47] N. Saleh, K. Ohashi, J. D. Thomson, and L. Chittka, "Facultative use of the repellent scent mark in foraging bumblebees: complex vs. simple flowers," Animal Behaviour, vol. 71, no. 4, pp. 847-854, 2006.

[48] N. Saleh, A. G. Scott, G. P. Bryning, and L. Chittka, "Distinguishing signals and cues: bumblebees use general footprints to generate adaptive behavior at flowers and nest," ArthropodPlant Interactions, vol. 1, pp. 119-127, 2007.

[49] B. Heinrich, Bumblebee Economics, Harvard University Press, Cambridge, Mass, USA, 1979.

[50] R. R. Sokal and F. J. Rohlf, Biometry, W.H. Freeman, New York, NY, USA, 1981

[51] T. Keasar, E. Rashkovich, D. Cohen, and A. Shmida, "Bees in two-armed bandit situations: foraging choices and possible decision mechanisms," Behavioral Ecology, vol. 13, no. 6, pp. 757-765, 2002.

[52] S. C. Stearns and P. Schmid-Hempel, "Evolutionary insights should not be wasted," Oikos, vol. 49, no. 1, pp. 118-125, 1987.

[53] A. Kacelnik and I. A. Todd, "Psychological mechanisms and the marginal value theorem: effect of variability in travel time on patch exploitation," Animal Behaviour, vol. 43, no. 2, pp. 313-322, 1992. 

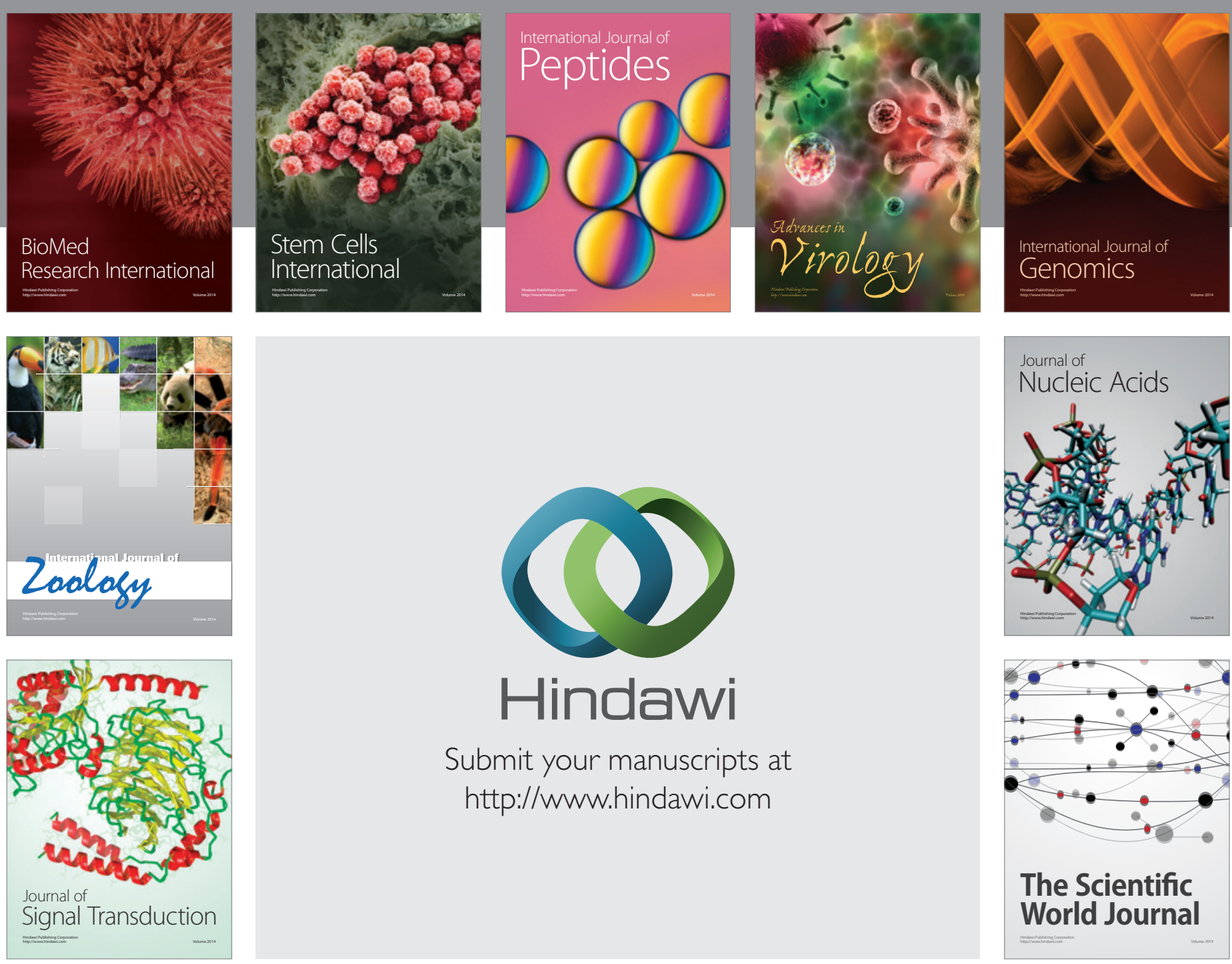

Submit your manuscripts at

http://www.hindawi.com
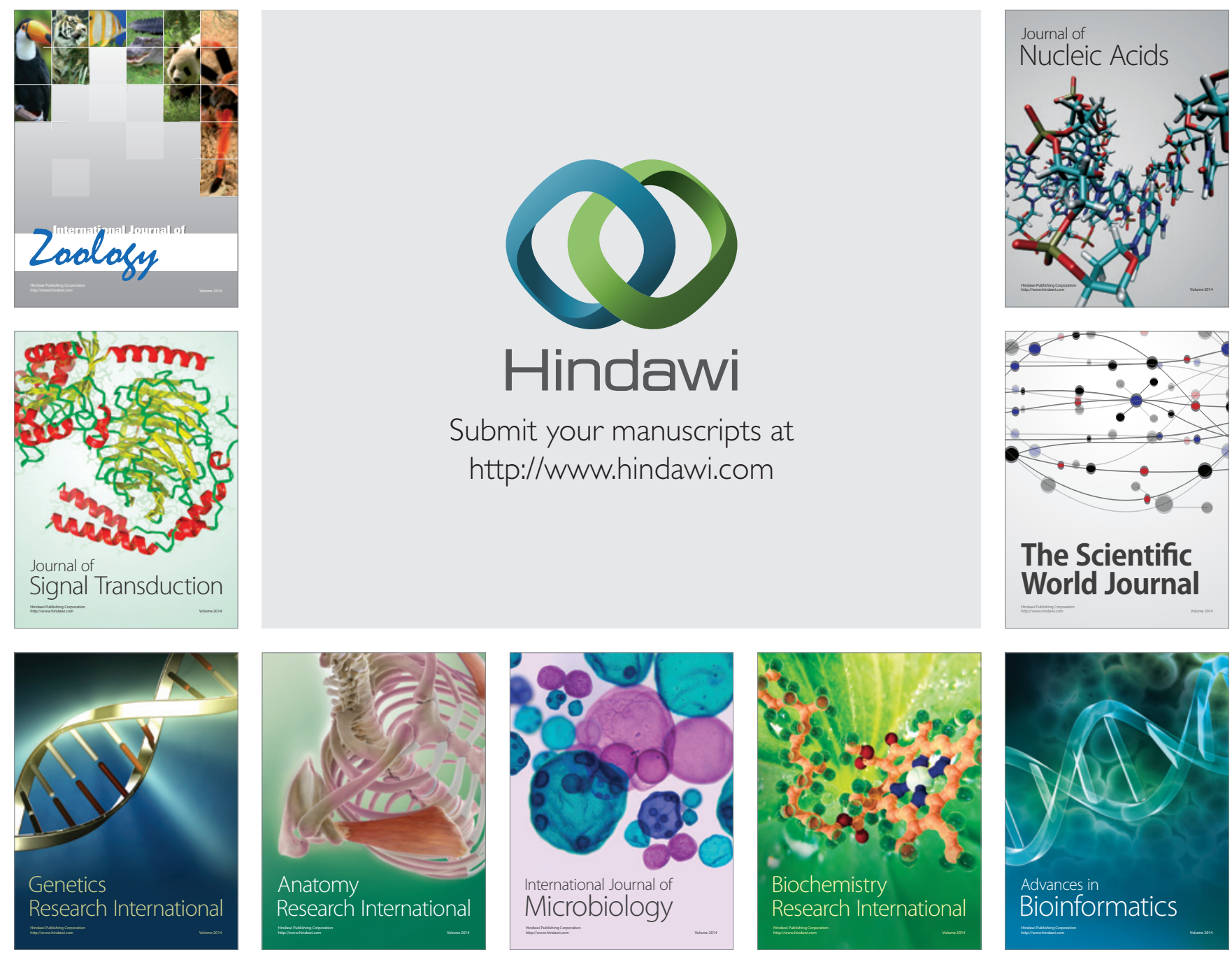

The Scientific World Journal
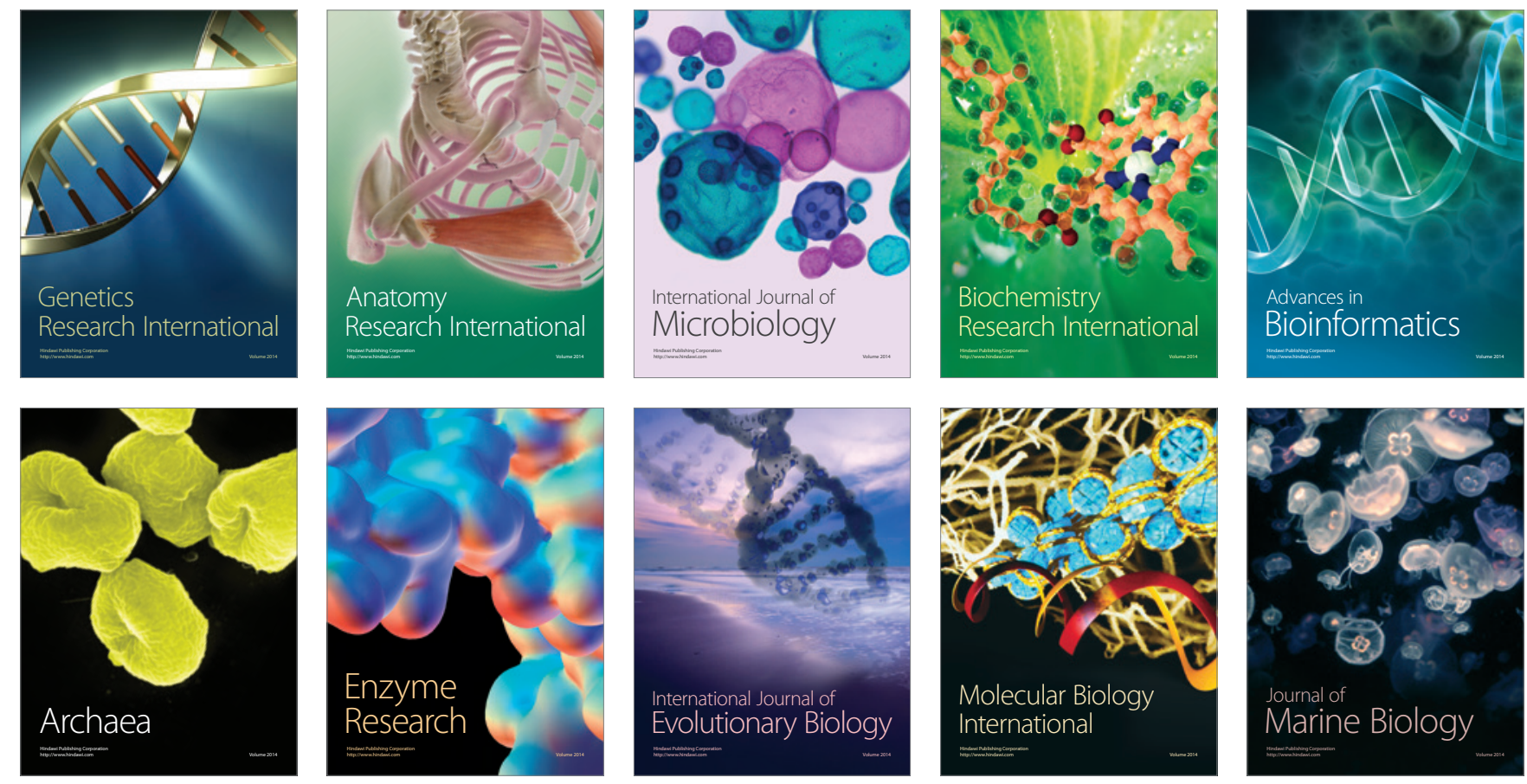